\title{
A new method for isolating turbulent states in transitional stratified plane Couette flow
}

\author{
J. R. TA Y L O R ${ }^{1} \dagger$, E. DEUSE B I O ${ }^{1}$, \\ C. P. CA ULFIEL D ${ }^{2,1}$ AND R. R. KER S W EL L \\ ${ }^{1}$ Department of Applied Mathematics and Theoretical Physics, \\ University of Cambridge, Centre for Mathematical Sciences, Willberforce Road, CB3 0WA, \\ Cambridge \\ 2 BP Institute, University of Cambridge, Madingley Road, CB3 0EZ, Cambridge \\ ${ }^{3}$ School of Mathematics, University of Bristol, BS8 1TW, Bristol
}

(Received ?; revised ?; accepted ?. - To be entered by editorial office)

We present a new adaptive control strategy to isolate and stabilize turbulent states in transitional, stably-stratified plane Couette flow in which the gravitational acceleration (non-dimensionalised as the bulk Richardson number $\mathrm{Ri}$ ) is adjusted in time to maintain the turbulent kinetic energy (TKE) of the flow. We demonstrate that applying this method at various stages of decaying stratified turbulence halts the decay process and allows a succession of intermediate turbulent states of decreasing energy to be isolated and stabilized. Once the energy of the initial flow becomes small enough, we identify a single minimal turbulent spot, and lower energy states decay to laminar flow. Interestingly, the turbulent states which emerge from this process have very similar time-averaged $R i$, but TKE levels different by an order of magnitude. The more energetic states consist of several turbulent spots, each qualitatively similar to the minimal turbulent spot. This suggests that the minimal turbulent spot may well be the lowest energy turbulent state which forms a basic building block of stratified plane Couette flow. The fact that a minimal spot of turbulence can be stabilized so that it neither decays nor grows opens up exciting opportunities for further study of spatiotemporally intermittent stratified turbulence.

\section{Introduction}

It is well-known that fluid flows with a linearly stable laminar state can still undergo a subcritical transition to turbulence and display a wealth of interesting, complex spatiotemporal dynamics which continues to defy understanding. For example, in plane Couette flow where a constant-density fluid of kinematic viscosity $\nu$ is sheared between two parallel boundaries a distance $2 h$ apart moving at velocity $\pm U$, there is a Reynolds number, $R e=U h / \nu=R e_{g} \simeq 323$, below which the laminar flow is a global attractor but turbulent spots can be triggered transiently (Bottin et al. 1997; Duguet et al. 2010). For $R e>R e_{g}$, the flow can permanently support spatiotemporal intermittency with coexistence of regions of turbulent and laminar flow (Lundbladh \& Johansson 1991; Tillmark \& Alfredsson 1992; Dauchot \& Daviaud 1995). The volume fraction occupied by turbulence increases with $R e$ until it fills the whole domain for $R e>R e_{t} \simeq 400$ (Duguet et al. 2010). Close to but above $R e_{g}$, there is a patterned regime where the turbulence arranges itself into regular stripes or bands obliquely oriented to the mean flow and separated by laminar regions (Prigent et al. 2002; Barkley \& Tuckerman 2005; Duguet et al.

$\dagger$ Email address for correspondence: jrt51@cam.ac.uk 
2010; Philip \& Manneville 2011; Manneville 2012; Duguet \& Schlatter 2013). This general picture is repeated across other shear flows undergoing a finite-amplitude transition: for example, certain regimes of Taylor-Couette flow (Coles 1965; Van Atta 1966; Prigent et al. 2002) and channel flow (Carlson et al. 1982; Alavyoon et al. 1986; Fukudome et al. 2009; Tsukahara et al. 2014). It also persists under the addition of extra physics such as rotation, Lorentz forces and stratification (Brethouwer et al. 2012; Deusebio et al. 2014).

Underpinning all this behaviour is the basic phenomenon of a localised finite-amplitude disturbance triggering the growth of a turbulent spot. These spots are never stable, either eventually decaying away if the underlying shear is too low $\left(R e<R e_{g}\right)$ or developing into a spatially-extended turbulent state such as a turbulent strip $\left(R e_{g}<R e<R e_{t}\right)$ or indeed the whole flow domain $\left(R e>R e_{t}\right)$. Observations and numerical calculations indicate that the evolution of a turbulent spot quickly takes on a universal structure independent of the initial disturbance. As a result, much work has been done to try to unravel the dynamics of this growth even though the very fact that the spot is continually evolving has made this difficult (Emmons 1951; Klingmann \& Alfredsson 1990; Carlson et al. 1982; Alavyoon et al. 1986; Henningson et al. 1987; Schumacher \& Eckhardt 2001).

Stratification introduces additional parameters, the Richardson number, Ri, which quantifies the relative importance of buoyancy compared to shear, and the Prandtl (or Schmidt) number, $\operatorname{Pr}=\nu / \kappa$, the ratio of the fluid viscosity to the scalar diffusivity. Here, we will consider only $\operatorname{Pr}=0.7$, characteristic of the diffusion of heat in air, while varying $R i$. Stable density stratification is common in geophysical flows. Despite the very high $R e$ associated with geophysical flows, turbulence is often highly intermittent as the de-stabilizing influence of vertical shear competes against the stabilizing influence of stratification (e.g. Mahrt 1999). Previous studies have examined the laminar-turbulent transition and intermittency in wall-bounded stratified shear flows (Flores \& Riley 2010; García-Villalba \& del Álamo 2011; Brethouwer et al. 2012; Deusebio et al. 2014, 2015). Figure 1 summarizes the simulations reported in Deusebio et al. (2015) with blue circles used to indicate simulations with spatiotemporal intermittency and red squares used to indicate fully turbulent flow.

Motivated by this, we use $R i$ as a control parameter to study spatiotemporal intermittency using direct numerical simulations (DNS) of stratified plane Couette flow. For simplicity, we consider a single $R e$, which is nearly three times larger than the critical value for unstratified plane Couette flow and $R i \in[0.02,0.2]$ : the baseline simulation with $R i=0.02$ is indicated by a filled blue circle in figure 1 . In the absence of stratification, flow at this $R e$ would be in the 'featureless' turbulence regime. However, stable stratification retards the transition process and the flow exhibits spatiotemporally intermittency in the form of patterned turbulence which will be described below. Note that most of the other simulations from Deusebio et al. (2015) used a relatively small box size and were unable to distinguish between patterned and irregular intermittent turbulence.

The paper is organised as follows. In section 2.1, we briefly describe the numerical setup and revisit one of the simulations reported in Deusebio et al. (2015) in an intermittent regime, which we use as a baseline simulation. In section 2.2 , we describe a series of decay simulations, each of which starts from an initial condition taken from the baseline simulation at $R i=0.02$. The decay simulations differ only in the new value of $R i>0.02$ subsequently imposed. Section 2.3 then describes a novel strategy to isolate and stabilize a turbulent spot by turning on the dynamic adaptation of $R i$ in the decay simulation for $R i=0.05$. The adaptive control procedure allows us to halt the decay process and isolate stripes and spots that characterise intermittent flows with a supercritical transition. Finally, we end with a brief summary and discussion in section 3 . 


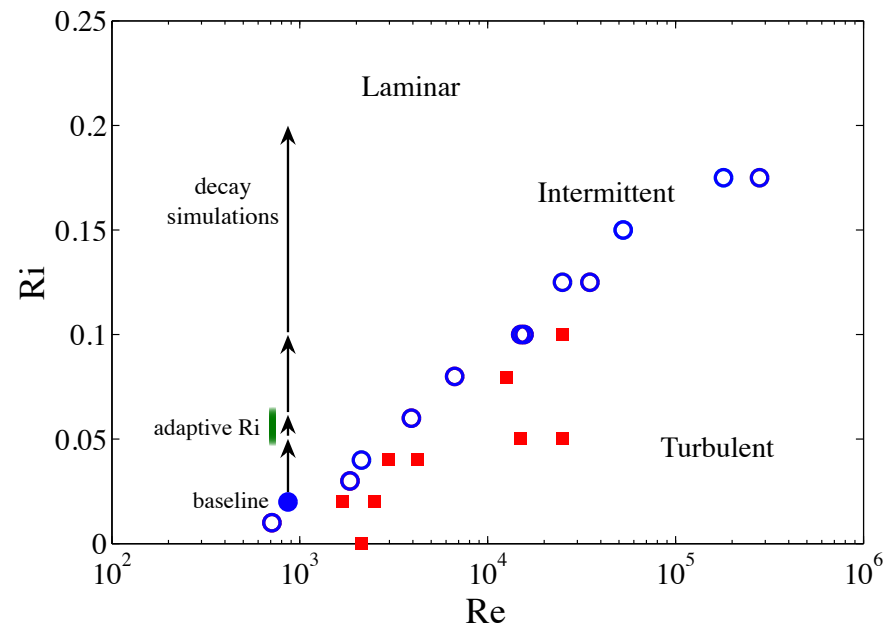

FiguRE 1. Flow regimes in stratified plane Couette flow as a function of Reynolds number, $R e$, and Richardson number, $R i$. All simulations use a Prandtl number $\operatorname{Pr}=0.7$. The simulations reported in Deusebio et al. (2015) are indicated with symbols. Blue circles denote simulations with spatiotemporal intermittency and red squares denote fully turbulent simulations. The solid blue circle is the baseline simulation described below in section 2.1. Arrows indicate an abrupt increase in $R i$ at the start of each decay simulation described in section 2.2 , and the green bar indicates the approximate range of $R i$ seen in the adaptive simulations $\mathrm{B}, \mathrm{C}$, and $\mathrm{D}$, described in section 2.3 .

\section{Results}

\subsection{Setup}

Stratified plane-Couette flow is bounded at the top and bottom by flat, rigid plates separated by a distance $2 h$. Here, $x, y$, and $z$ will be used to denote the streamwise, wall-normal, and spanwise directions, respectively, following the standard convention for Couette and channel flow. The plates move in opposite directions with constant velocity $\mathbf{u}= \pm U \hat{\mathbf{x}}$. The walls are held at a fixed temperature, $\theta$, such that the temperature of the upper wall is $2 T$ larger than the temperature of the lower wall. Here, we consider the following nondimensional incompressible Boussinesq equations with a linear equation of state:

$$
\begin{aligned}
\frac{\partial \mathbf{u}}{\partial t}+\mathbf{u} \cdot \nabla \mathbf{u} & =-\nabla p+\operatorname{Ri\theta } \hat{\mathbf{y}}+\frac{1}{R e} \nabla^{2} \mathbf{u}, \\
\frac{\partial \theta}{\partial t}+\mathbf{u} \cdot \nabla \theta & =\frac{1}{\operatorname{RePr}} \nabla^{2} \theta \\
\nabla \cdot \mathbf{u} & =0
\end{aligned}
$$

where

$$
R e:=U h / \nu, \quad R i:=|\mathbf{g}| \alpha T h / U^{2}, \quad \operatorname{Pr}:=\nu / \kappa,
$$

$\mathrm{g}=-g \hat{\mathbf{y}}$ is the acceleration due to gravity which acts in the $-y$ direction, $\nu$ is the kinematic viscosity, $\kappa$ is the thermal diffusivity, $\alpha$ is the thermal expansion coefficient, and $p$ is the nondimensional pressure.

Periodic boundary conditions are applied in $x$ and $z$. The numerical code uses a pseudospectral method in $x$ and $z$ and second order finite differences to calculate derivatives in the $y$ direction. The timestepping algorithm is a mixed implicit/explicit scheme using 
the 3rd order Runge-Kutta and Crank-Nicolson methods. Further details of the problem configuration and numerical method can be found in Deusebio et al. (2015).

One of the simulations reported by Deusebio et al. (2015) is chosen as our 'baseline' simulation (filled blue circle in Figure 1). This simulation has $R e=865, R i=0.02$, and $\operatorname{Pr}=0.7$. Although $R e$ is among the lowest considered by Deusebio et al. (2015), it is still sufficiently high to be in the 'fully turbulent' regime for unstratified plane-Couette flow

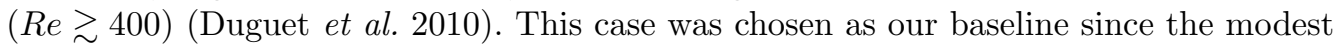
resolution allow us to use a very large domain in the streamwise and spanwise directions, with $L_{x}=64 \pi h$ and $L_{z}=32 \pi h$. The simulation uses 1024 gridpoints in the $x$ and $z$ directions and 64 points in $y$, with gridpoints clustered near both walls. As discussed by Deusebio et al. (2015), the large domain size reduces temporal intermittency of the flow and provides robust statistics.

Panel 1 of Figure 2(b) shows the streamwise velocity at $y=-0.5 h$, the horizontal plane halfway between the lower wall and the centerline. Turbulent and laminar regions develop in inclined bands reminiscent of those seen at lower Reynolds numbers in transitional plane-Couette flow (Prigent et al. 2002). Deusebio et al. (2015) described a method for identifying local turbulent and laminar regions and defined the 'turbulent fraction', $\gamma$, as the fraction of the total domain occupied by turbulent flow. For sufficiently large Re and small $R i$, the flow is fully turbulent with $\gamma=1$ (red squares in Figure 1). For sufficiently small $R e$ and large $R i$, intermittent flow is seen with $0<\gamma<1$ (circles in Figure 1). For the baseline simulation, $\gamma=0.64$ (solid circle in Figure 1).

\subsection{Decay simulations}

In the first set of simulations, we varied the strength of the stable stratification by abruptly increasing $R i$. Each simulation was initialized from the baseline simulation described above with $R e=865$ and $R i=0.02$ with a state obtained at statistical equilibrium. The decay simulations are indicated in Figure 1 as vertical arrows ending at the new Richardson number. For convenience, $t=0$ will correspond to the time when $R i$ is abruptly increased. Since $R i$ multiplies the buoyancy term in the non-dimensional vertical momentum equation, increasing $R i$ is equivalent to increasing the gravitational acceleration, i.e. heavy fluid becomes heavier and light fluid becomes lighter.

Figure 2(a) shows the time evolution of the turbulent kinetic energy, TKE $:=\left\langle\mathbf{u}^{\prime} \cdot \mathbf{u}^{\prime}\right\rangle / 2$, where $\langle\cdot\rangle$ denotes a volume average and primes denote a departure from this average, i.e. $\mathbf{u}^{\prime}:=\mathbf{u}-\langle\mathbf{u}\rangle$. In four cases with $R i \geqslant 0.05$, the TKE decays in time at an approximately exponential rate, and the rate of decay increases with $R i$. Eventually all simulations with $R i \geqslant 0.05$ reach a fully laminar state. When $R i=0.03$ and $R i=0.04$, the TKE decays during a transient period and partially recovers, but remains below the initial value. For comparison, a continuation of the baseline case with $R i=0.02$ is also shown.

The decay process does not proceed uniformly in space, but instead turbulence persists in localized 'pockets' contained within receding turbulent bands, qualitatively similar to what has been seen in previous simulations of decaying unstratified turbulence (Manneville 2011). Figure 2(b) shows four snapshots of the streamwise velocity at $y=-0.5 h$ in the decay simulation with $R i=0.05$. The corresponding times are indicated using dots in Figure 2(a). Highly localized patches of turbulence can be seen at times labeled 3 and 4 , while the rest of the flow is nearly laminar.

\subsection{Adaptive Ri simulations}

In order to examine further the influence of stratification on the flow near the laminarturbulent transition, we have developed a procedure using the Richardson number, $R i$,

as an adaptive control parameter. To our knowledge, this is the first time that $R i$ has 
(a)

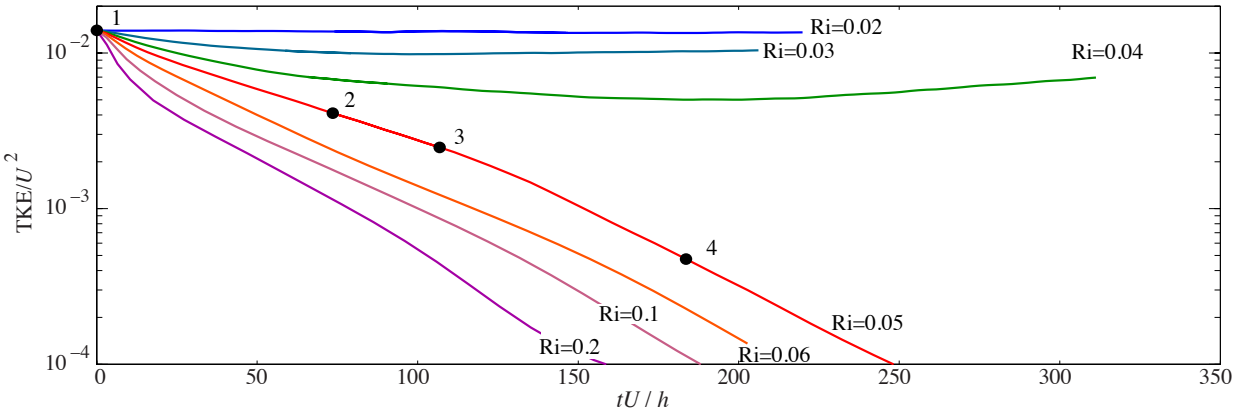

(b)
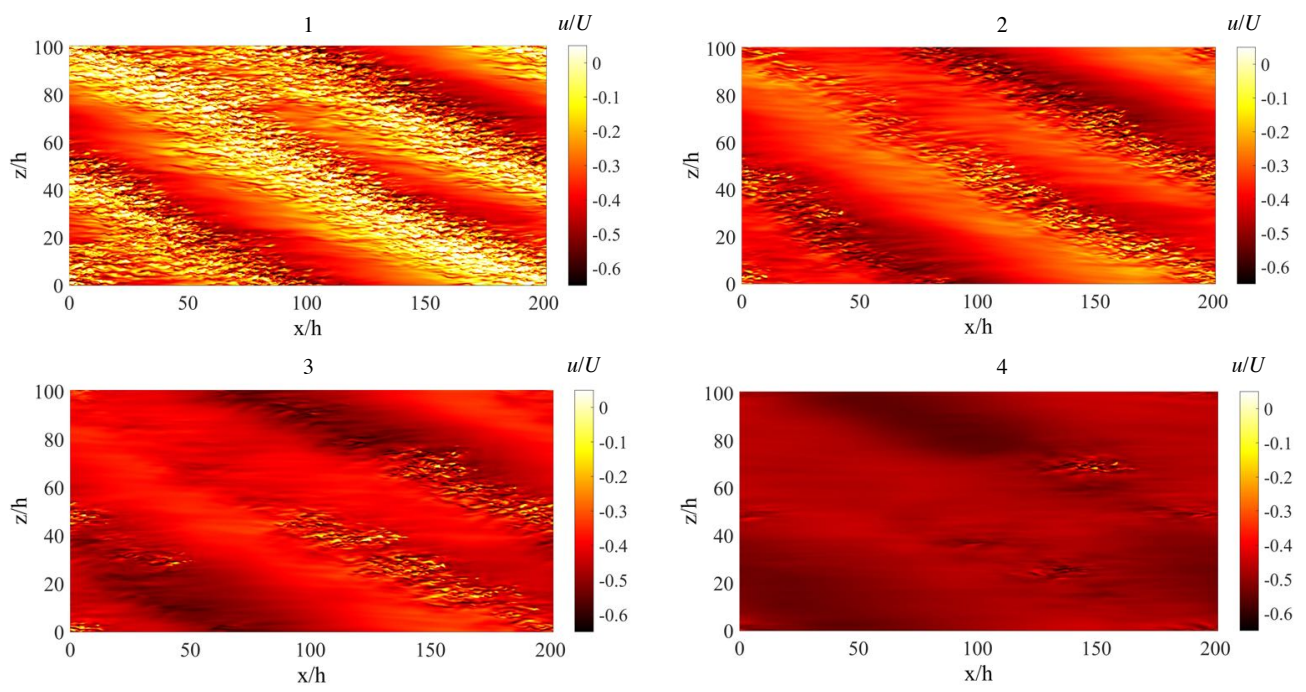

FigurE 2. (a) Time series of the turbulent kinetic energy (TKE) in the decay simulations. Each simulation starts from the same initial state labeled 1. The Richardson number in each simulation is labeled. (b) Horizontal slices of the streamwise velocity at $y=-0.5 h$ at various times in the simulation with $R i=0.05$. The corresponding times are indicated with dots and numbered in panel (a). The first time (panel 1) shows the velocity in the baseline simulation that was used to initialize the decay simulations. In this simulation spatiotemporal intermittency takes the form of a repeating pattern of bands (or stripes) of turbulence inclined with respect to the spanwise direction.

been used to control the level of turbulence in a stratified flow. The procedure allows us to isolate turbulent states progressively closer to the re-laminarization boundary. The adaptive procedure changes $R i$ based on the rate of change of TKE. Let $t_{0}$ correspond to the time $M$ timesteps before the current time, $t$, and let $\operatorname{TKE}\left(t_{0}\right)$ and $\operatorname{TKE}(t)$ be the TKE at these times. The TKE decay timescale, $\tau$, between times $t_{0}$ and $t$ is

$$
\tau:=\frac{-\left(t-t_{0}\right)}{\ln \left(\frac{T K E(t)}{T K E\left(t_{0}\right)}\right)}
$$

and the Richardson number, $R i(t)$, is then set as

$$
R i(t):=R i\left(t_{0}\right)-c \frac{t-t_{0}}{\tau}
$$


where $\operatorname{Ri}\left(t_{0}\right)$ was the previous value at $t_{0}$, and $c$ is a control parameter that will be discussed later. The adaptive $R i$ procedure acts to maintain a roughly constant value of the TKE. The turbulent fraction and TKE level are sensitive to Ri (Deusebio et al. $2015)$. During periods where TKE decreases in time $(\tau>0)$, the adaptive procedure will decrease $R i$ which has the ultimate effect of increasing the TKE, thus leading to the possibility of 'controlling' the turbulence at a nontrivial level. Mathematically, if $R i$ was adjusted every time step, equations (2.4) and (2.5) are a finite difference approximation to imposing the extra dynamical constraint

$$
\frac{d}{d t}\left[R i(t)+c \ln \left(\frac{T K E}{T K E_{0}}\right)\right]=0,
$$

to the Boussinesq equations and $T K E_{0}$ is an arbitrary constant energy. Note that $c=0$ recovers the Boussinesq equations with constant $R i$.

In the adaptive Ri simulations, we updated $R i$ using (2.5) every $M=20$ timesteps and set $c=0.1$. The value of $M$ was chosen to reduce the computational cost of this procedure while ensuring that $R i$ is adjusted sufficiently often to keep pace with any change in the TKE decay rate. In practice, the time between $R i$ adjustment steps is always less than one advective unit, $t-t_{0}<h / U$. The sensitivity of the results to the choice of $c$ will be examined later. We have used the adaptive $R i$ procedure to isolate turbulent states during various stages of the decay process. We saved 3D flow fields at various times during the decay simulation with $R i=0.05$, indicated by dots in Figure $3(\mathrm{a})$. Each of these was then used as an initial condition for an adaptive $R i$ simulation. To ensure continuity, $R i$ was initialized to 0.05 in the adaptive $R i$ simulations.

Time series of the TKE from the adaptive $R i$ simulations are shown in dashed lines in Figure 3(a). The decay simulation with $R i=0.05$ is shown for reference (solid line). The simulations with adaptive $R i$ are each labeled with a letter (A-G). In simulations labeled A-D, the TKE continues to decrease at the start of the adaptive simulation, but soon reaches a quasi-steady state. Simulations initialized later in the decay process have quasi-steady states with lower TKE. Time series of $R i$ in the adaptive simulations is shown in Figure 3(b). In all cases $R i$ reaches a quasi-steady state after an initial drop mirroring the TKE. Interestingly, the quasi-steady value of $R i$ in cases $\mathrm{B}, \mathrm{C}$, and D falls in the same range, $R i \simeq 0.05-0.06$, despite very different values of the TKE in these simulations. Simulation A has a smaller quasi-steady value of $R i \simeq 0.03$. For context, the approximate range of $R i$ in the quasi-steady state for simulations $\mathrm{B}, \mathrm{C}$, and D is indicated as a green band in Figure 1.

Simulation D is the lowest level of TKE that we have been able to reach using this procedure. In simulations E-G, the flow nearly relaminarises during the adaptive $R i$ simulation. In this regime, $R i$ oscillates and eventually becomes negative (Figure $3 \mathrm{~b}$ ), while the flow develops into a series of streamwise-independent rolls (not shown) corresponding closely to slightly supercritical, sheared Rayleigh-Bénard convection (Clever et al. 1977; Kelly 1977). There are dramatic differences in the character of the flow and the value of the steady state $R i$ in simulations $\mathrm{D}$ and $\mathrm{E}$, implying that the flow in simulation D is close to the re-laminarization boundary.

The streamwise velocity at $y=-0.5 h$ is shown for four adaptive Ri simulations in Figure 4. Each snapshot corresponds to the end of the adaptive Ri simulation, as labeled in Figure 3(a). Simulation A was initialized directly from the baseline simulation (magenta dot) with $R i=0.05$ and the TKE decreases slightly at the start of the simulation. Turbulent/laminar bands are still prominent features of simulation A, and the laminar regions are more coherent than in the baseline simulation (compare Figure $2 \mathrm{~b}$ panel 1 and Figure 4 panel A). In the other extreme, simulation D has a single turbulent spot. 
(a)

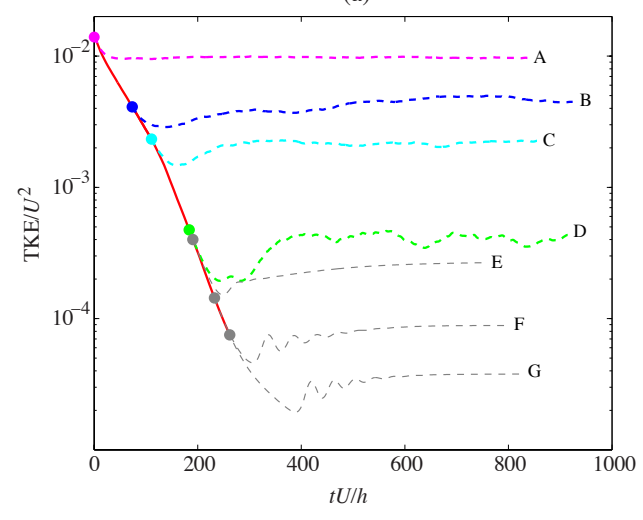

(b)

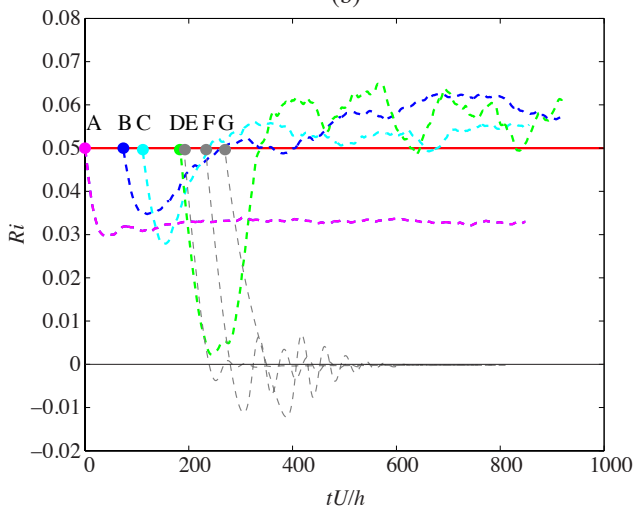

FigurE 3. (a) Time series of the turbulent kinetic energy (TKE) in the adaptive Ri simulations. The simulations are initialized from various times in the $R i=0.05$ decay simulations as indicated by colored dots. (b) Time series of the Richardson number, $R i$, for each of the adaptive Ri simulations using the control scheme described in the text. The solid red line indicates $R i=0.05$ from the decay simulation. The start time of each simulation is indicated with a colored dot, and the colors and labels are the same as in panel (a)
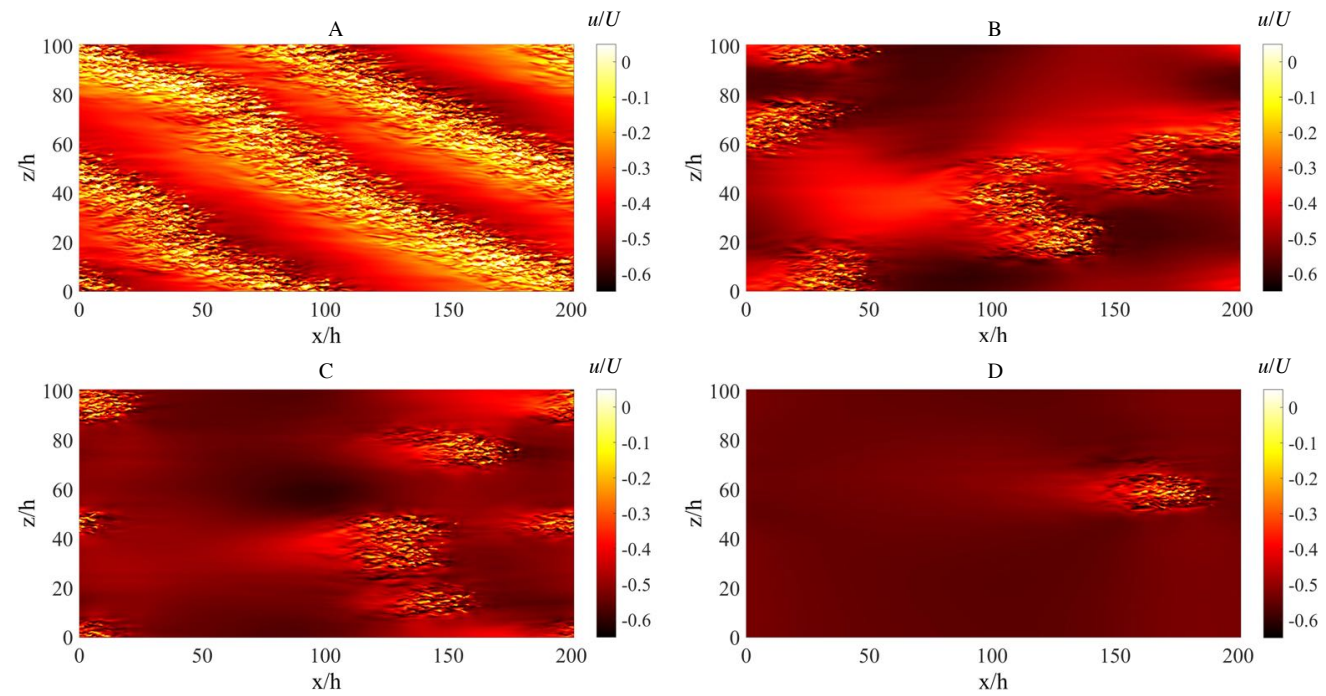

FiguRE 4. Horizontal slices of the streamwise velocity at $y=-0.5 h$ at the end of the adaptive Ri simulations. Labels are the same as in Figure 3.

This spot persists throughout the adaptive Ri simulation, slowly migrating around the domain. Simulations B and C have multiple turbulent spots resembling the one seen in simulation D. In simulation B some of the spots appear to join together with some indications of short inclined bands of turbulence.

The turbulent structures in the isolated turbulent spot are qualitatively similar to the structures in the turbulent stripes. Figure 5 shows vertical slices of the temperature field from simulations A and D illustrating these two regimes. Turbulence in the stripes and spot spans the gap between the lower and upper plates, and is inclined in the direction of plate motion. Small regions with an unstable density arrangement (heavy over light) occur in the turbulent patch. Features reminiscent of developing Kelvin-Helmholtz billows 

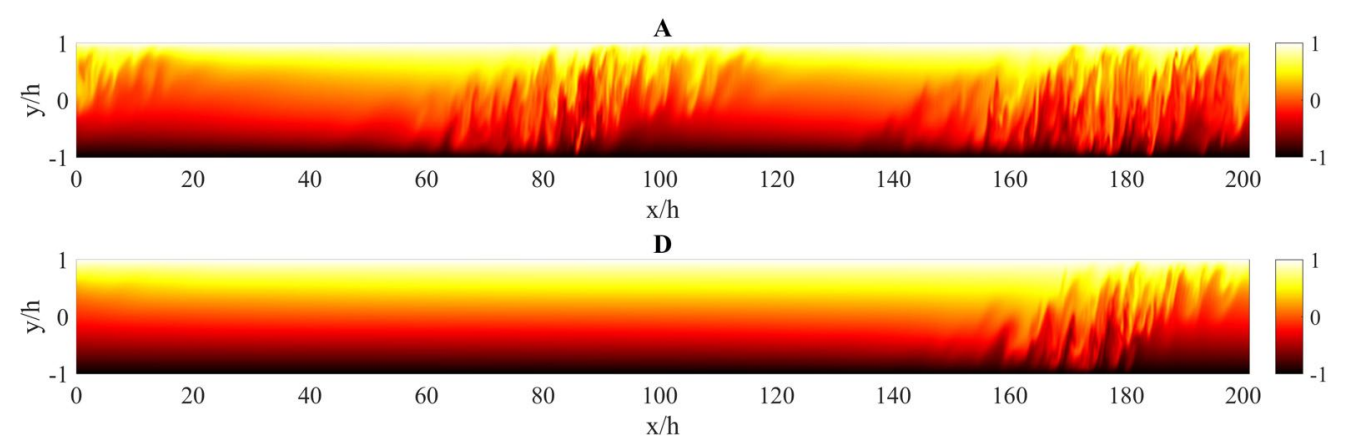

FiguRE 5. Nondimensional temperature $(\theta / T)$ on vertical slices passing through $z=60 h$ for simulations A (top panel) and D (bottom panel). The simulation names are the same as in Figures 3 and 4 .

appear on the flanks of the turbulent patches, for example between $60 \leqslant x / h \leqslant 80$ in simulation $\mathrm{A}$ and $150 \leqslant x / h \leqslant 170$ in simulation $\mathrm{D}$ (note that the aspect ratio is highly stretched).

To examine the sensitivity of our results to details of the adaptive $R i$ procedure, we continued simulation D (exhibiting a single turbulent spot) with various values of the adjustment coefficient, $c$, including a case with $c=0$ when $R i$ is held fixed. Time series of the TKE and $R i$ are shown in Figure 6 . When $c=1$, the TKE remains close to constant in time, but $R i$ exhibits large, high frequency oscillations. In contrast, when $R i$ is held fixed $(c=0)$, the TKE undergoes large low-frequency oscillations in time. In this uncontrolled simulation the turbulent spot first grows, splits into two spots, and one of these spots decays (Figure 6, right panels). The remaining spot looks remarkably similar to the controlled spot from which the simulation was started, more than 400 advective time units earlier (compare panels I and III). This suggests that the controlled spot is at least qualitatively representative of the uncontrolled system. The intermediate values $c=0.1$ and $c=0.01$, represent a trade-off between larger oscillations in $R i$ and TKE, respectively.

A natural question to ask is whether the captured turbulent spot obtained using the adaptive control procedure is sensitive to the initial conditions. That is, does the flow retain some 'memory' of the simulation with decaying stratified turbulence from which it was started? To address this question, we ran an additional simulation initialized with a localized 'seed' following the procedure of Lagha \& Manneville (2007). Specifically, we started with the velocity from a simulation of unstratified plane Couette flow at the same Reynolds number in the featureless turbulence regime. The initial velocity was then formed by multiplying the unstratified velocity field by a Gaussian function of $x$ and $z$,

$$
\mathbf{u}(\mathbf{x}, t=0)=\mathbf{u}_{R i=0}(\mathbf{x}) e^{-\left(x^{2}+z^{2}\right) / S},
$$

where $S=2 h^{2}$. Note that this seed is significantly smaller than the turbulent spot seen in simulation D. The seed was allowed to develop with $R i=0$ for 35 advective time units, after which point, the adaptive procedure was started with $c=0.1$. Although the Richardson number was started from $R i=0$, it converged towards approximately the same value seen in simulations $\mathrm{B}, \mathrm{C}$, and $\mathrm{D}$, and the flow developed into a single turbulent spot that appeared extremely similar in structure to the one in simulation D. This result implies that the turbulent spot is not sensitive to the initialization procedure. When the adaptive procedure was started without first allowing the seed to grow, the increase in stratification caused the flow to re-laminarize. This supports the idea that the turbulent 

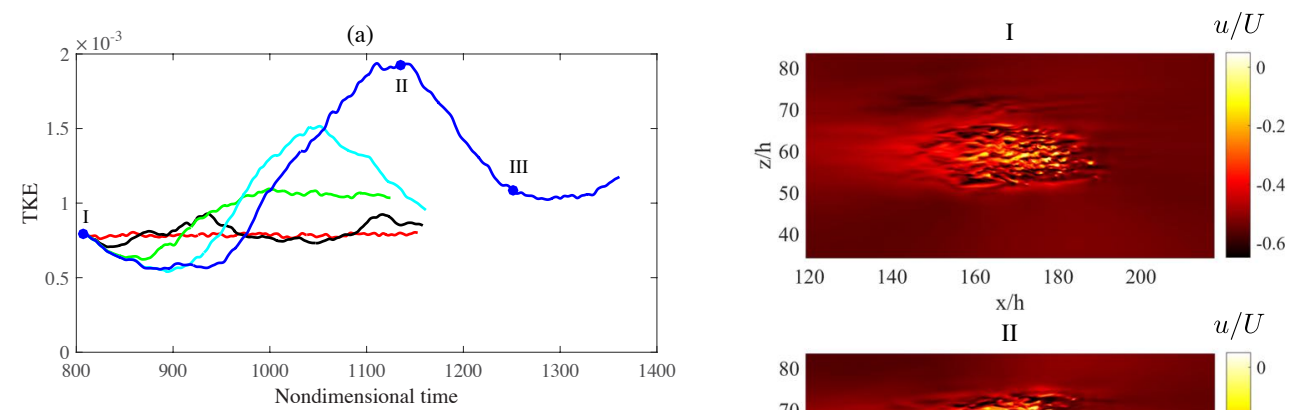

(b)
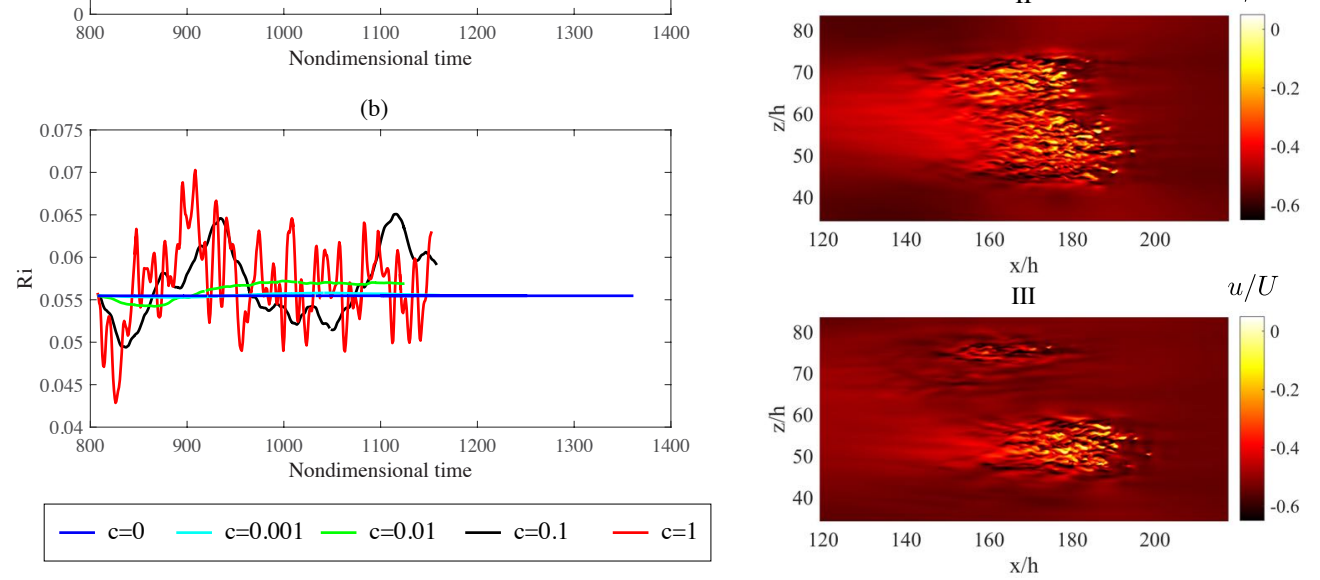

Figure 6. Dependence of the TKE (a) and Richardson number (b) to the choice of the coefficient, $c$, in Eq. 2.5. Each simulation is started from the end of simulation D with a single controlled turbulent spot. Visualizations of the streamwise velocity from an uncontrolled simulation $(c=0)$ are shown for the times indicated in blue dots in panel (a).

spot captured in simulation D is close to the minimal energy turbulent attractor, the lowest energy turbulent state that can be maintained using the adaptive $R i$ procedure.

\section{Discussion}

We have examined intermittent stratified plane-Couette flow using direct numerical simulations. While the Reynolds number was kept fixed, the Richardson number, $R i$, was used as a control parameter, allowing us to probe the dynamics of intermittent stratified turbulence in this geometry. We have considered two sets of simulations. In the first set of simulations, $R i$ is abruptly increased relative to a control simulation. For sufficiently large values of $R i$, the flow re-laminarizes. However, in these simulations, localized patches of turbulence persist into the decay period until the flow becomes fully laminar. This result is qualitatively similar to Manneville (2011) who found breakup of turbulent bands in decaying unstratified plane-Couette flow.

Next, we describe a new method to isolate flow structures at very low turbulent energy using $R i$ as an adaptive control parameter. The method adjusts $R i$ based on the time rate of change in the turbulent kinetic energy (TKE), increasing $R i$ when the TKE is increasing and lowering $R i$ when the TKE is decreasing. The adaptive control procedure acts to stabilize the flow close to the initial energy level. By starting the adaptive procedure at various times during a simulation of decaying stratified turbulence, we are able to isolate a variety of low energy flow structures including spots and short stripes of turbulence.

When the Richardson number changes through the control procedure introduced here, 
the buoyancy of the fluid changes everywhere in the domain instantaneously. It is difficult to imagine a means to change the density of a real fluid in a similar way by changing its temperature or salinity. However, a change in buoyancy could be interpreted as a change in the gravitational acceleration. An analogue laboratory experiment would measure the change in TKE within a given time window and accelerate the entire Couette flow apparatus up or down so as to change the fluid buoyancy instantaneously everywhere throughout the flow. Although it would be difficult to implement this system in a practical laboratory experiment, it provides a physical interpretation of the control procedure.

The lowest energy turbulent structure that we are able to identify using the adaptive control procedure consists of a single isolated turbulent spot in an otherwise laminar flow. Qualitatively, this stabilized spot is very similar to the spots seen previously in unstratified transitional flows and it seems reasonable to assume that they are closely related. Studying this stabilized spot then offers an exciting opportunity to probe the dynamics seen. However, confirming a connection is not as simple as examining the $c \rightarrow 0$ limit because the fluctuations in energy become larger as $c$ decreases until eventually the spot becomes uncontrollable at some small but finite $c$ (see figure 6 ).

The scheme to adapt $R i$ based on the time rate of change of $T K E$ is somewhat arbitrary, and other choices could be made. For example, it might be possible to control a turbulent spot in unstratified flow using the Reynolds number as a control parameter. One could use this technique to explore the dynamics of multiple interacting controlled spots. For example, if a simulation were initialised with several isolated turbulent spots, would they coalesce into stripes and eventually form patterns? If so, do the properties of the turbulent spot provide insight into properties of patterned turbulence such as the width of the turbulent bands?

Another interesting issue is how the stabilized spot in the controlled system - the Boussinesq equations augmented by the constraint (2.6)) with $R i(t)$ now a dynamic variable - is related to the laminar-turbulent boundary or 'edge' (Itano \& Toh 2001; Skufca et al. 2006) in the uncontrolled Boussinesq equations with, say, the required fixed value of $R i$ defined as $\overline{R i}$, the long time average of $R i(t)$ (a reasonable but not unique choice to link the controlled system with variable $R i(t)$ and the uncontrolled system with fixed $R i$ ). In the controlled system, the spot is a stable low-energy state and must therefore sit 'above' the edge (i.e. not in the laminar basin of attraction) but presumably still close to it. The edge for the controlled system has an extra dimension in phase space compared to that for the uncontrolled system due to $R i(t)$ being an extra dynamic variable. Projecting the edge in the controlled system down onto the hyperplane with fixed $R i=\overline{R i(t)}$ might produce a good estimate for the edge in the uncontrolled system provided the fluctuations in $R i(t)$ are not too large compared to $\overline{R i(t)}$. However, it is unclear where the projected stabilized spot solution will land in phase space relative to the uncontrolled system edge: i.e. it could be inside, outside or even on it, and so the control procedure is not a direct competitor to bisection-based edge tracking (Itano \& Toh 2001; Skufca et al. 2006) which endeavours to work on the edge.

In this paper we have only considered a single Reynolds number, $R e=U h / \nu=865$. Although this Reynolds number is large enough to support fully-developed turbulence in the unstratified limit, it is much smaller than typical values in geophysical and industrial flows. One consequence of the moderate Reynolds number is that the Richardson number associated with intermittent flow is also modest. Deusebio et al. (2015) found intermittent behaviour at much higher Reynolds numbers for sufficiently large Richardson number, which is also consistent with previous work (e.g. Brethouwer et al. 2012; García-Villalba $\&$ del Álamo 2011; Flores \& Riley 2010). The fate of the laminar-turbulent transition boundary in the limit as $R e \rightarrow \infty$ is one of the most important open problems in stratified 
turbulence. Knowing whether there is a finite 'critical' value of $R i$ above which turbulence cannot persist as $R e \rightarrow \infty$ would be of great use in parameterizing turbulence and mixing in ocean, atmosphere, and climate models.

One of the difficulties with answering this question is the rapid increase in computational cost with increasing $R e$. Very close to the transition boundary, where stratification suppresses energetic turbulence and localizes turbulent patches, the flow might be more accessible to DNS. However, this accessible region narrows in parameter space as $R e \rightarrow \infty$ (Deusebio et al. 2015) making it difficult to locate the transition boundary. Here, too our adaptive control technique might be helpful since the long-time average of $\operatorname{Ri}(t)$ needed to maintain a low perturbation energy level as $R e$ increases should be a good predictor for this boundary.

The EPSRC grant EP/K034529/1 entitled 'Mathematical Underpinnings of Stratified Turbulence' is gratefully acknowledged for supporting the research presented here. We thank Paul Linden, Stuart Dalziel, and the MUST team for many helpful discussions and helpful comments from three anonymous referees.

\section{REFERENCES}

Alavyoon, F., Henningson, D. S. \& Alfredsson, P. H. 1986 Turbulent spots in plane Poiseuille flow-flow visualization. Phys. Fluids 29 (4), 1328-1331.

Barkley, D. \& Tuckerman, L. S. 2005 Computational study of turbulent laminar patterns in Couette flow. Phys. Rev. Lett. 94 (1), 014502.

Bottin, S., Dauchot, O. \& Daviaud, F. 1997 Intermittency in a locally forced plane Couette flow. Phys. Rev. Lett. 79 (22), 4377-4380.

Brethouwer, G., Duguet, Y. \& Schlatter, P. 2012 Turbulent-laminar coexistence in wall flows with Coriolis, buoyancy or Lorentz forces. J. Fluid Mech. 704, 137-172.

Carlson, D. R., Widnall, S. E. \& Peeters, M. F. 1982 A flow-visualization study of transition in plane Poiseuille flow. J. Fluid Mech. 121, 487-505.

Clever, R.M., Busse, F.H. \& Kelly, R.E. 1977 Instabilities of longitudinal convection rolls in Couette flow. Zeitschrift für angewandte Mathematik und Physik ZAMP 28 (5), 771-783.

Coles, D. 1965 Transition in circular Couette flow. J. Fluid Mech. 21 (03), 385-425.

Dauchot, O. \& Daviaud, F. 1995 Finite amplitude perturbation and spots growth mechanism in plane Couette flow. Phys. Fluids 7 (2), 335-343.

Deusebio, E., Brethouwer, G., Schlatter, P. \& Lindborg, E. 2014 A numerical study of the unstratified and stratified Ekman layer. J. Fluid Mech. 755, 672-704.

Deusebio, E., Caulfield, C. P. \& Taylor, J. R. 2015 The intermittency boundary in stratified plane Couette flow. J. Fluid Mech. 781, 298-329.

Duguet, Y. \& Schlatter, P. 2013 Oblique laminar-turbulent interfaces in plane shear flows. Phys. Rev. Lett. 110 (3), 034502.

Duguet, Y., Schlatter, P. \& Henningson, D. S. 2010 Formation of turbulent patterns near the onset of transition in plane Couette flow. J. Fluid Mech. 650, 119.

Emmons, H.W. 1951 The laminar-turbulent transition in a boundary layer - Part I. J. Aero. Sci. 18 (7).

Flores, O. \& Riley, J. J. 2010 Analysis of turbulence collapse in stably stratified surface layers using direct numerical simulation. Boundary Layer Meteorol. 129 (2), 241-259.

Fukudome, K., Iida, O. \& NaGano, Y. 2009 The mechanism of energy transfer in turbulent Poiseuille flow at very low Reynolds number. In Proc. 6th Intl Symp. on Turbulence and Shear Flow Phenomena, pp. 471-476.

García-Villalba, M. \& Del Álamo, J. C. 2011 Turbulence modification by stable stratification in channel flow. Phys. Fluids 23 (4), 045104. 
Henningson, D., Spalart, P. \& Kim, J. 1987 Numerical simulations of turbulent spots in plane Poiseuille and boundary-layer flow. Phys. Fluids 30 (10), 2914-2917.

Itano, T. \& Tон, S. 2001 The dynamics of bursting process in wall turbulence. J. Phys. Soc. Japan 70, 703-716.

Kelly, RoBert E 1977 The onset and development of Rayleigh-Bénard convection in shear flows: a review. Physicochemical hydrodynamics 1, 65-79.

Klingmann, B. G. B. \& Alfredsson, P. H. 1990 Turbulent spots in plane Poiseuille flow measurements of the velocity field. Phys. Fluids A 2 (12), 2183-2195.

Lagha, M. \& Manneville, P. 2007 Modeling of plane Couette flow: I. large scale flow around turbulent spots. Phys. Fluids 19 (9), 094105.

Lundbladi, A. \& Johansson, A. V. 1991 Direct simulation of turbulent spots in plane Couette flow. J. Fluid Mech. 229, 499-516.

Mahrt, L. 1999 Stratified atmospheric boundary layers. Boundary-Layer Meteor. 90 (3), 375396.

Manneville, P. 2011 On the decay of turbulence in plane Couette flow. Fluid Dyn. Res. 43 (6), 065501.

Manneville, P. 2012 On the growth of laminar-turbulent patterns in plane Couette flow. Fluid Dyn. Res. 44 (3), 031412.

Philip, J. \& Manneville, P. 2011 From temporal to spatiotemporal dynamics in transitional plane Couette flow. Phys. Rev. E 83 (3), 036308.

Prigent, A., Grégoire, G., Chaté, H., Dauchot, O. \& van SaArloos, W. 2002 Largescale finite-wavelength modulation within turbulent shear flows. Phys. Rev. Lett. 89 (1), 014501.

SChumaCher, Jörg \& ECKhardt, Bruno 2001 Evolution of turbulent spots in a parallel shear flow. Physical Review E 63 (4), 046307.

SkufcA, J.D., Yorke, J.A. \& Eckhardt, B. 2006 Edge of chaos in parallel shear flow. Phys. Rev. Lett. 96, 174101.

Tillmark, N. \& Alfredsson, P. H. 1992 Experiments on transition in plane Couette flow. J. Fluid Mech. 235, 89-102.

Tsukahara, T., Seki, Y., Kawamura, H. \& Tochio, D. 2014 Dns of turbulent channel flow at very low Reynolds numbers. arXiv preprint arXiv:1406.0248.

Van Atta, C. 1966 Exploratory measurements in spiral turbulence. J. Fluid Mech. 25 (03), $495-512$. 